\title{
Conformally Invariant Differential Operators on Minkowski Space and Their Curved Analogues
}

\author{
Michael G. Eastwood ${ }^{1}{ }^{\star}$ and John W. Rice ${ }^{2}$ \\ 1 Department of Pure Mathematics, University of Adelaide, G.P.O. Box 498, Adelaide, South \\ Australia 5001 \\ 2 School of Mathematics, Flinders University, Bedford Park, South Australia 5042
}

\begin{abstract}
This article describes the construction of a natural family of conformally invariant differential operators on a four-dimensional (pseudo-)Riemannian manifold. Included in this family are the usual massless field equations for arbitrary helicity but there are many more besides. The article begins by classifying the invariant operators on flat space. This is a fairly straightforward task in representation theory best solved through the theory of Verma modules. The method generates conformally invariant operators in the curved case by means of Penrose's local twistor transport.
\end{abstract}

\section{Introduction}

Special relativistic field equations are those which are invariant under the Poincaré group. The Poincare invariance of Maxwell's equations may be regarded as the starting point for the special theory of relativity. Maxwell's equations and other massless field equations can, however, be made to exhibit $[2,7]$ an even larger invariance group, namely the conformal group of Minkowski space. Our first objective in this article is to describe all conformally invariant differential operators on Minkowski space.

Our second objective is to exhibit precise analogues of these differential operators on space-times which are not flat. For this, the sense of the analogy is the important point since there is no longer a transitive group of conformal transformations acting on a curved space-time; invariance under a conformal group becomes meaningless. However, for differential operators depending on a choice of metric there is the concept of invariance under conformal rescaling of the metric, i.e. replacing the metric $g_{a b}$ with a metric $\hat{g}_{a b}=\Omega^{2} g_{a b}$ for $\Omega$ a nowhere-vanishing function. If $D$ is a differential operator corresponding to $g_{a b}$ then denote by $\hat{D}$ the operator corresponding to the rescaled metric $\hat{g}_{a b}$. One allows tensors, etc., to be rescaled by powers of $\Omega$, so that if we decide to put $\hat{\phi}=\Omega^{r} \phi$, then we say that $\phi$ is

* S.E.R.C. Advanced Fellow and Flinders University Visiting Research Fellow 
being considered as a conformal density of weight $r$. We say that a (metric dependent) differential operator is conformally invariant, in the sense of rescaling, if $\hat{D} \hat{\phi}=\Omega^{s} D \phi$, when $\phi$ is given some conformal weight $r$. If $D$ is regarded as acting on conformal densities of weight $r$ to give conformal densities of weight $s$, then this equation may be rewritten as $\hat{D} \hat{\phi}=\widehat{D \phi}$. Consider, for example, the massless free field equations of helicity $n / 2$. Using the notation of $[22,27]$, these may be written $\nabla^{A A^{\prime}} \phi_{A^{\prime} B^{\prime} \cdots D^{\prime}}=0$, where the field $\phi_{A^{\prime} B^{\prime} \cdots D^{\prime}}$ is symmetric in its spinor indices (of which there are $n$ in number) and is also conformally weighted of weight -1 . When $g_{a b}$ is rescaled, the Levi Civita connnection changes according to [24,27],

$$
\hat{\nabla}_{A A^{\prime}} \phi_{B^{\prime}}=\nabla_{A A^{\prime}} \phi_{B^{\prime}}-r_{A B^{\prime}} \phi_{A^{\prime}},
$$

where $\Upsilon_{a}=\Omega^{-1} \nabla_{a} \Omega$. Thus,

$$
\hat{\nabla}_{E E^{\prime}} \hat{\phi}_{A^{\prime} B^{\prime} \cdots D^{\prime}}=\hat{\nabla}_{E E^{\prime}}\left[\Omega^{-1} \phi_{A^{\prime} B^{\prime} \cdots D^{\prime}}\right]=\Omega^{-1}\left[\nabla_{E E^{\prime}} \phi_{A^{\prime} B^{\prime} \cdots D^{\prime}}-(n+1) \Upsilon_{E\left(E^{\prime}\right.} \phi_{\left.A^{\prime} B^{\prime},,, D^{\prime}\right)}\right] \text {. }
$$

and, consequently,

$$
\hat{\nabla}^{A A^{\prime}} \hat{\phi}_{A^{\prime} B^{\prime} \cdots D^{\prime}}=\hat{g}^{a e} \hat{\nabla}_{E E^{\prime}} \hat{\phi}_{A^{\prime} B^{\prime} \cdots D^{\prime}}=\Omega^{-2} g^{a e} \hat{\nabla}_{A^{\prime} B^{\prime} \cdots D^{\prime}}=\Omega^{-3} \nabla^{A A^{\prime}} \phi_{A^{\prime} B^{\prime} \cdots D^{\prime}} .
$$

This demonstrates the well-known (e.g. $[22,27]$ ) conformal invariance of these equations. Perhaps a more interesting example is provided by $\square+R / 6$, where $\square$ is the Laplacian (i.e. the wave operator in the Lorentzian case) and $R$ is the scalar curvature. It too acts from conformal densities of weight -1 to those of weight -3 . There are higher order conformally invariant operators which have the same form, i.e. which reduce to an operator invariant under the conformal group in the flat case, and appear as the flat operator corrected by curvature terms in general. For example, $\square^{2}$ admits such a conformally invariant modification [15] when acting on functions (weight 0 ) to produce 4-forms (weight -4).

One might ask for the most general rescaling invariant operator, but the question is ambiguous, admitting several formulations even for zeroth order operators, i.e. tensors (e.g. [30] versus [16]). One could reasonably ask for those polynomial expressions in the Levi Civita connection, the metric, and its inverse (thus including curvature) which turn out to be invariant under conformal rescaling. However, this seems to be a rather ad hoc requirement, and it would be more satisfying to find a more definitive and elementary requirement. For Minkowski space invariance under the conformal group is such a requirement. Moreover, for conformally flat spacetimes this global conformal invariance relates quite directly to the local rescaling invariance as follows.

Suppose $D$ is a differential operator on compactified Minkowski space [24] invariant under the group of global conformal transformations. Suppose $M$ is a conformally flat space-time and choose on $M$ an open set together with a choice of metric thereon which is flat. Choose a similar open set in Minkowski space and map one to the other by an isometry. In this way one can attempt to transfer the differential operator $D$ over to $M$ and it remains to show that this is a consistent procedure. Here the point is that any two choices are related by a local conformal map from an open subset of Minkowski space onto another. Liouville's result (e.g. [9]) that any such local transformation is the restriction of a global conformal automorphism of compactified Minkowski space now ensures consistency. 
In fact it will turn out that the rescaling invariant operators derived from those invariant under the group of conformal automorphisms of Minkowski space have a similar form to those examples at the beginning of this introduction, i.e. a leading order term comprising some simple composition of Levi Civita connections, together with lower order terms with various components of curvature and their covariant derivatives as coefficients. We will show that these same formulae define conformally invariant operators (in the sense of conformal rescaling) on a general space-time. These are the "curved analogues" in the title of this article.

This paper is very much inspired by Penrose's twistor theory. Two preliminary observations are taken directly from this theory. The first is that any reasonable definition of conformal invariance for a real (pseudo-) Riemannian manifold will work equally well for all signatures as well as in the complexification (see e.g. $[20,12]$ ) where there is no longer any distinction between Riemannian, Lorentzian, etc.. Thus, the space-times in this article will be complex. The second observation is the basis of twistor theory $[23,25,26,28]$, namely that complexified compactified Minkowski space $\mathbb{M}$ can be regarded as the Grassmannian of 2-planes in $\mathbb{C}^{4}$ whence the global conformal transformations of $\mathbb{M}$ are $4-1$ covered by $S L(4, \mathbb{C})$ acting on $\mathbb{M}$ via the self representation on $\mathbb{C}^{4}$.

In Sect. 1 the flat space problem is reduced to a question in representation theory. With the introduction of Verma modules in Sects. 2 and 3, the problem is solved in Sects. 4 and 5. The method of construction used in Sect. 5 is designed so that it generalizes to the curved case. Following a suggestion of Roger Penrose, this is accomplished in Sect. 6.

We should point out that the representation theory that we use is, by modern standards, fairly elementary. There is much more sophisticated machinery which can be brought to bear and which demolishes the problem rather quickly (see $[4,5]$ where more general results are to be found). Thus, this paper must be considered as partly expository but, in addition to considerations of sledge-hammers versus nuts, our approach is constrained by the desire to obtain the curved version in Sect. 6 . The main tool of Sect. 6 is the local twistor connection [26,28]. This is the 4dimensional version of Cartan's conformal connection. Using this more general notion, Rob Baston [1] has succeeded in placing the arguments of this article in a much more general setting.

Conversations with Rob Baston and Rod Gover have been very helpful.

\section{Formulation of the Problem}

In order to be properly defined, conformal transformations require "points at infinity" to be added to Minkowski space. Indeed, if $(t, x, y, z)$ are Minkowski coordinates and

$$
\|(t, x, y, z)\|^{2}=t^{2}-x^{2}-y^{2}-z^{2},
$$

then the so-called "inversion in the origin", $p \mapsto p /\|p\|^{2}$, is conformal but is undefined on the light cone of the origin. The remedy is to compactify Minkowski space as explained, for example, in [24]. The result is a closed manifold with conformal structure on which the possibly singular conformal transformations of Minkowski 
space, such as inversion, act as genuine conformal automorphisms. Similar comments apply in the complex picture. It is the complex picture which we shall adopt from now on. Thus, all constructions are in the holomorphic category, i.e. manifolds are complex manifolds, tensors are holomorphic tensors, and so on.

As indicated in the introduction, complexified compactified Minkowski space $\mathbb{M}$ may be identified with the Grassmannian $G r_{2}\left(\mathbb{C}^{4}\right)$ of 2-planes in $\mathbb{C}^{4}$, and therefore as a homogeneous space for $S L(4, \mathbb{C})$. Specifically, $\mathbb{M}=G / P$, where $G=S L(4, \mathbb{C})$ and $P$ is the subgroup consisting of those matrices of the form

$$
\left(\begin{array}{llll}
* & * & * & * \\
* & * & * & * \\
0 & 0 & * & * \\
0 & 0 & * & *
\end{array}\right) .
$$

The theory to be explained in this article applies generally for $G$ a complex semisimple Lie group and $P$ a parabolic subgroup, i.e. one for which the quotient $G / P$ is compact. Actually, it is slightly more convenient and slightly more general to take $G$ to be the (reductive) group $G L(4, \mathbb{C}$ ). Until Sect. 3, however, the discussion is general and $G$ can be any complex Lie group with $P$ any complex subgroup.

A homogeneous vector bundle [3] on $G / P$ is, by definition, induced by a finite dimensional representation of $P$, say $\rho: P \rightarrow \operatorname{Aut}(E)$. The vector bundle is obtained by factoring the trivial bundle $G \times E$ on $G$ by the action of $P$ given by

$$
(\alpha, e) \mapsto\left(\alpha p^{-1}, \rho(p) e\right) \text { for } p \in P, \alpha \in G, e \in E .
$$

Equivalently, its sections are given locally by $E$-valued holomorphic functions $f$ on $G$ satisfying $\rho(p) f(\alpha p)=f(\alpha)$ for $p \in P, \alpha \in G$. $G$ acts on such local sections by means of $(g . f)(\alpha)=f\left(g^{-1} \alpha\right)$ for $g, \alpha \in G$. All tensor fields, for example, may be regarded as sections of homogeneous vector bundles since the tangent bundle is induced from the Adjoint action of $P$ on $\mathfrak{g} / \mathfrak{p}$, where $\mathfrak{g}$ and $\mathfrak{p}$ are the Lie algebras of $G$ and $P$ respectively. In Sect. 3 we will show that spinor fields on $\mathbb{M}$ may also be viewed as sections of appropriate homogeneous bundles. In what follows we shall use $E$ to denote the homogeneous bundle as well as the representation space of $\rho$.

Given two such homogeneous vector bundles $E$ and $F$, consider the (holomorphic) differential operators from $E$ to $F$. Since such operators act on local sections, it makes sense to require that they be invariant under the action of $G$ above. These will be called invariant differential operators. The exterior derivative $d: \Omega^{p} \rightarrow \Omega^{p+1}$ from $p$-forms to $(p+1)$-forms is always invariant in this sense. The problem is to classify all such invariant operators.

A differential operator of order $k$ is the same as a vector bundle homomorphism $D: J^{k} E \rightarrow F$, where $J^{k} E$ is the $k^{\text {th }}$ associated jet bundle of $E$ (e.g. [29]). Its fibre at $P \in G / P$ consists of germs of sections of $E$ modulo those that vanish at $P$ to order $k+1$. If $f$ vanishes to that order then so does $p \cdot f$ for any $p \in P$. Hence the fibre over $P$ carries a finite dimensional representation of $P$ from which $J^{k} E$ is reconstructed as a homogeneous vector bundle (in the same way as any homogeneous bundle is induced from the representation of $P$ on its fibre over $P \in G / P)$. As before, we denote the representation of $P$ by $J^{k} E$ also. If $D: J^{k} E \rightarrow F$ is a homomorphism of $P$ representations, then it induces an invariant homomorphism of the corresponding 
vector bundles, i.e. an invariant operator. Conversely, all invariant operators arise in this way. Consequently, the classification problem becomes the task of understanding the representations $J^{k} E$ followed by the purely algebraic problem of determining the $P$-homomorphisms $J^{k} E \rightarrow F$. Clearly the first part of this proposal involves not only the representation of $P$ on $E$ but also the ambient group $G$, whereas the second part concerns only the representation theory of $P$. In fact, it turns out that the approach we adopt for the first part (in the following section) greatly eases the second task if the embedding of $P$ in $G$ is not discarded (see Sect. 4).

\section{Dual Formulation via Verma Modules}

This section is concerned, in particular, with an algebraic method of constructing the jet representation $J^{k} E$. In fact, more precisely, $J^{k} E$ will only be displayed as a Lie algebra representation of $\mathfrak{p}$.

For all $k$ there is a surjective $p$-homomorphism $J^{k+1} E \rightarrow J^{k} E$, and we can form the projective limit

$$
J^{\infty} E \rightarrow \cdots \rightarrow J^{k+1} E \rightarrow J^{k} E \rightarrow J^{k-1} E \rightarrow \cdots \rightarrow J^{1} E \rightarrow E .
$$

As a simple example consider the case where $G=\mathbb{C}, P=\{0\}$, and $\rho$ is the trivial representation on $\mathbb{C}$. Evidently, the above system of jets is

$$
\mathbb{C}[[z]] \rightarrow \cdots \rightarrow \mathbb{C}[z] / z^{k+2} \rightarrow \mathbb{C}[z] / z^{k+1} \rightarrow \mathbb{C}[z] / z^{k} \rightarrow \cdots \rightarrow \mathbb{C}[z] / z^{2} \rightarrow \mathbb{C} .
$$

Here $\mathbb{C}[[z]]$ denotes the formal power series in the indeterminate $z$ and in general $J^{\infty} E$ may be regarded as the formal power series expansions of sections of $E$. This system is dual to

$$
\mathbb{C}[d / d z] \supset \cdots \supset \mathbb{C}_{k+1}[d / d z] \supset \mathbb{C}_{k}[d / d z] \supset \mathbb{C}_{k-1}[d / d z] \supset \cdots \supset \mathbb{C}_{1}[d / d z] \supset \mathbb{C},
$$

where $\mathbb{C}_{k}[d / d z]$ denotes the space of polynomials of degree $\leqq k$ in the indeterminate $d / d z$. Observe that $\mathbb{C}[d / d z]$ is the universal enveloping algebra $[8,19]$ of the Lie group $\mathbb{C}$ filtered by degree in the usual way. This example generalizes at once to the case where $G$ is an arbitrary complex Lie group but with $P$ and $\rho$ still trivial. Then the universal enveloping algebra $\mathfrak{A}(\mathfrak{g})$ filtered by degree clearly has as its dual the space of infinite jets of functions on $G$ at the identity. In order to incorporate a subgroup $P$ and a representation $\rho: P \rightarrow \operatorname{Aut}(E)$, first consider the $\mathfrak{U}(\mathfrak{g})$-module $\mathfrak{U}(\mathfrak{g}) \otimes_{\mathbb{C}} E^{*}$, where $\mathfrak{g}$ acts trivially on $E^{*}$, the dual of $E$. This corresponds to the trivial vector bundle $G \times E$ on $G$. Using $\rho$ to pass down to a homogeneous bundle on $G / P$ evidently corresponds to factoring $\mathfrak{U}(\mathfrak{g}) \otimes_{\mathbb{C}} E^{*}$ by the left $\mathfrak{U}(\mathfrak{g})$-submodule generated by $\left\{p \otimes e-1 \otimes \rho^{*}(p) e\right\}$ for $p \in \mathfrak{p}, e \in E^{*}$ (and where $\rho^{*}: \mathfrak{p} \rightarrow$ End $\left(E^{*}\right)$ also denotes the derivative of $\left.\rho^{*}: P \rightarrow \operatorname{Aut}\left(E^{*}\right)\right)$. This induced $\mathfrak{H}(\mathfrak{g})$-module shall be denoted $V(\rho)$ and is called the Verma module associated to $\mathfrak{g}, \mathfrak{p}$, and $\rho$. Often the term Verma module is reserved for the case where $E$ is one-dimensional $[31,3,8]$ but we shall use it also to refer to the general concept above. An excellent review of the one-dimensional case and in investigation of the general case is to be found in [21]. The grading of $\mathfrak{U}(\mathfrak{g})$ induces a grading of $V(\rho)$ :

$$
V(\rho) \supset \cdots \supset V_{k+1}(\rho) \supset V_{k}(\rho) \supset V_{k-1}(\rho) \supset \cdots \supset V_{1}(\rho) \supset V_{0}(\rho)=E^{*},
$$

with each level preserved by $\mathfrak{p}$. It is a matter of pure thought to check that taking 
duals gives

$$
J^{\infty} E \rightarrow \cdots \rightarrow J^{k+1} E \rightarrow J^{k} E \rightarrow J^{k-1} E \rightarrow \cdots \rightarrow J^{1} E \rightarrow E
$$

as $\mathfrak{U}(\mathfrak{p})$-modules.

Now if $J^{k} E \rightarrow F$ is a $P$-homomorphism for representations $\rho$ and $\sigma$ on $E$ and $F$ respectively, then it induces $J^{j+k} E \rightarrow J^{j} F$ for all $j$ and so $J^{\infty} E \rightarrow J^{\infty} F$. Dually, this corresponds to a homomorphism of $\mathfrak{U}(\mathfrak{g})$-modules $V(\sigma) \rightarrow V(\rho)$. These observations may be reversed to conclude that the search for invariant differential operators is equivalent to the search for homomorphisms between Verma modules. The order of the corresponding operator is the least $k$ for which the image of $F^{*}=V_{0}(\sigma)$ lies in $V_{k}(\rho)$. The composition

$$
V_{0}(\sigma) \rightarrow V_{k}(\rho) \rightarrow V_{k}(\rho) / V_{k-1}(\rho)
$$

is then, by virtue of the jet exact sequence [29],

$$
0 \rightarrow \odot^{k} \Omega^{1} \otimes E \rightarrow J^{k} E \rightarrow J^{k-1} E \rightarrow 0,
$$

dual to the composition $\bigodot^{k} \Omega^{1} \otimes E \rightarrow J^{k} E \rightarrow F$, which provides the symbol of the operator [29]. Here $\odot$ means the symmetric tensor power and $\Omega^{1}$ is the homogeneous bundle induced from the coAdjoint representation of $P$ on $(\mathfrak{g} / \mathfrak{p})^{*}$. From this one can begin with a prospective symbol and ask whether it lifts to be the symbol of an invariant differential operator (cf. [17]). However, we shall adopt a more direct approach to determining the homomorphisms $V(\sigma) \rightarrow V(\rho)$.

In general, the Verma modules $V(\rho)$ are complicated owing to the complicated nature of $\mathfrak{u}(\mathfrak{g})$. However, when $\mathfrak{g}$ is semisimple and $\mathfrak{p}$ is parabolic a great deal can be said. In this case one can always write $\mathfrak{g}=\mathfrak{p}+\mathfrak{q}$ (a vector space direct sum of Lie subalgebras) whence, by the Poincaré-Birkhoff-Witt theorem [19], $V(\rho)=$ $\mathfrak{U}(\mathfrak{q}) \otimes_{\mathbb{C}} E^{*}$, with grading induced by that of $\mathfrak{U}(\mathfrak{q})$. The action of $\mathfrak{g}$ is then a result of $(\mathrm{i})$ the action of $\mathfrak{q}$ on $\mathfrak{U}(\mathfrak{q})$ alone and (ii) the action of $\mathfrak{p}$ obtained by using the commutation relations between $\mathfrak{p}$ and $\mathfrak{q}$ to commute elements of $\mathfrak{p}$ past $\mathfrak{U}(\mathfrak{q})$ whereupon they strike $E^{*}$ according to $\rho^{*}$. Although these are still quite complicated objects, the homomorphisms between Verma modules constructed from irreducible representations are partially known $[21,4,5]$ and indeed completely known in case $\mathfrak{p}$ is also minimal, i.e. a Borel subalgebra.

\section{The Structure of Verma Modules for Minkowski Space}

This section describes the Verma modules $V(\rho)$ for Minkowski space $\mathbb{M}=G / P$, where $\rho$ is an irreducible representation of $P$. This description follows a general scheme valid for any parabolic subgroup of a semisimple or reductive Lie group. Any unjustified statements made in this section are directly verifiable for Minkowski space. The general approach may be found in $[3,19,21]$ for example.

To fix notation we shall label the generators of $\mathrm{gl}(4, \mathbb{C})$ according to the scheme

$$
\left(\begin{array}{llll}
h_{1} & X_{1} & x_{2} & x_{4} \\
Y_{1} & h_{2} & x_{1} & x_{3} \\
y_{2} & y_{1} & h_{3} & X_{2} \\
y_{4} & y_{3} & Y_{2} & h_{4}
\end{array}\right) .
$$


Then $\mathfrak{g}=\mathfrak{p}+\mathfrak{q}$, where $\mathfrak{q}$ is the subalgebra consisting of matrices of the form

$$
\left(\begin{array}{cccc}
0 & 0 & 0 & 0 \\
0 & 0 & 0 & 0 \\
y_{2} & y_{1} & 0 & 0 \\
y_{4} & y_{3} & 0 & 0
\end{array}\right) .
$$

Notice that $q$ is Abelian (a further simplification as compared with the general case), whence $\mathfrak{U}(\mathfrak{q})$ is simply the polynomial algebra $\mathbb{C}\left[y_{1}, y_{2}, y_{3}, y_{4}\right]$. Thus, for $\rho: P \rightarrow \operatorname{Aut}(E)$ a finite dimensional representation of $P$,

$$
V(\rho)=\mathbb{C}\left[y_{1}, y_{2}, y_{3}, y_{4}\right] \otimes_{\mathbb{C}} E^{*},
$$

with grading determined by polynomial degree.

Let $L$ denote the subgroup of $P$ consisting of matrices of the form

$$
\left(\begin{array}{llll}
* & * & 0 & 0 \\
* & * & 0 & 0 \\
0 & 0 & * & * \\
0 & 0 & * & *
\end{array}\right)
$$

with corresponding Lie algebra $l(=\mathfrak{g l}(2, \mathbb{C}) \times \mathfrak{g l}(2, \mathbb{C}))$. In general $L$ is called a Levi factor of the parabolic $P$. Every finite dimensional irreducible representation of $P$ is derived from a finite dimensional irreducible representation of $L$ by means of $P \rightarrow L$. These representations may be classified by either highest or lowest weights, using the usual "raising" and "lowering" arguments with $X_{1}, X_{2}$ as raising operators and $Y_{1}, Y_{2}$ as lowering operators. The result is that homogeneous bundles on $\mathbb{M}$ are in $1-1$ correspondence with the symbols $(a, b \mid c, d)$ for integers $a \leqq b$ and $c \leqq d$, meaning that the corresponding representation is on a vector space $E$ containing a lowest weight vector $\delta$ with

$$
h_{1} \delta=a \delta, \quad h_{2} \delta=b \delta, \quad h_{3} \delta=c \delta, \quad h_{4} \delta=d \delta .
$$

This notation agrees with that in $[13,14]$ where the description is given in terms of Young tableau. In the notation of [10], $(a, b \mid c, d)$ is the spinor bundle

$$
\mathcal{O}(\overbrace{A B \cdots D}^{d-c})(\overbrace{E^{\prime} F^{\prime} \cdots H^{\prime}}^{b-a}[d][-a]^{\prime} .
$$

The dual representation $E^{*}$ is therefore constructed from a highest weight vector $\alpha$ with

$$
h_{1} \alpha=-a \alpha, \quad h_{2} \alpha=-b \alpha, \quad h_{3} \alpha=-c \alpha, \quad h_{4} \alpha=-d \alpha .
$$

It is evident that $V(a, b \mid c, d)=\mathbb{C}\left[y_{1}, y_{2}, y_{3}, y_{4}\right] \otimes E^{*}$ is itself a highest weight module for $\mathfrak{U}(\mathfrak{g})$ in the sense that it contains a highest weight vector, namely $1 \otimes \alpha$ (which from now on will just be denoted by $\alpha$ ) annihilated by $X_{1}, X_{2}, x_{1}, x_{2}, x_{3}, x_{4}$ and that $V(a, b \mid c, d)$ is generated by applying the lowering operators $Y_{1}, Y_{2}, y_{1}, y_{2}, y_{3}, y_{4}$.

Now suppose that $D: V(e, f \mid g, h) \rightarrow V(a, b \mid c, d)$ is a $\mathfrak{U}(\mathrm{g})$-homomorphism. Let $\beta$ denote the highest weight vector generating $V(e, f \mid g, h)$. Then $D(\beta)$ must be a highest weight vector in $V(a, b \mid c, d)$, i.e. it must be annihilated by $X_{1}, X_{2}, x_{1}, x_{2}, x_{3}, x_{4}$ and provide a joint eigenvector for $h_{1}, h_{2}, h_{3}, h_{4}$ (though it does not necessarily generate 
$V(a, b \mid c, d)$ under lowering). Conversely, if $\gamma$ is a highest weight vector in $V(a, b \mid c, d)$ with weight given by

$$
h_{1} \gamma=-e \gamma, \quad h_{2} \gamma=-f \gamma, \quad h_{3} \gamma=-g \gamma, \quad h_{4} \gamma=-h \gamma,
$$

Then $D: V(e, f \mid g, h) \rightarrow V(a, b \mid c, d)$ may be defined by mapping $\beta \mapsto \gamma$ and applying lowering operators to determine $D$ on the rest of $V(e, f \mid g, h)$.

Thus, the search for invariant differential operators $(a, b \mid c, d) \rightarrow(e, f \mid g, h)$ is entirely equivalent to the search for highest weight vectors in $V(a, b \mid c, d)$. Note that since $x_{2}, x_{3}$, and $x_{4}$ can be generated from commutators of $X_{1}, X_{2}$, and $x_{1}$ (e.g. $\left.\left[X_{1}, x_{1}\right]=x_{2}\right)$, it suffices to find weight vectors annihilated by $X_{1}, X_{2}$, and $x_{1}$. In fact, it is easy to check that a weight vector $\gamma$ say

$$
h_{1} \gamma=-e \gamma, \quad h_{2} \gamma=-f \gamma, \quad h_{3} \gamma=-g \gamma, \quad h_{4} \gamma=-h \gamma
$$

annihilated by $X_{1}$ and $X_{2}$ corresponds to a possible symbol i.e. to a $P$-module homomorphism,

$$
\bigodot^{k} \Omega^{1} \otimes(a, b \mid c, d) \rightarrow(e, f \mid g, h) .
$$

Thus, $x_{1} \gamma$ may be regarded as the obstruction to this being a genuine symbol, i.e. the symbol of a genuine invariant differential operator. This also shows that the symbol of an invariant operator between irreducible bundles determines the operator. Using that $\Omega^{1}=(0,1 \mid-1,0)$, it is at this stage a tractable task, with knowledge of the Clebsch-Gordan coefficients for $\mathfrak{g l}(2, \mathbb{C})$, to determine all symbols and thus all invariant operators. This task is simplified, however, by the use of central characters as explained in the next section.

This section ends by way of illustration with a few invariant operators. Consider first the Verma module,

$$
V(0,0 \mid 0,0)=\mathbb{C}\left[y_{1}, y_{2}, y_{3}, y_{4}\right] \alpha,
$$

where $h_{1} \alpha=h_{2} \alpha=h_{3} \alpha=h_{4} \alpha=0$. The symbol $\Omega^{1} \otimes(0,0 \mid 0,0) \rightarrow \Omega^{1}$ corresponds to the weight vector $y_{1} \alpha$. Note that

$$
\begin{aligned}
& h_{1} y_{1} \alpha=\left[h_{1}, y_{1}\right] \alpha+y_{1} h_{1} \alpha=\left[h_{1}, y_{1}\right] \alpha=0, \\
& h_{2} y_{1} \alpha=\left[h_{2}, y_{1}\right] \alpha+y_{1} h_{2} \alpha=\left[h_{2}, y_{1}\right] \alpha=-y_{1} \alpha, \\
& h_{3} y_{1} \alpha=\left[h_{3}, y_{1}\right] \alpha+y_{1} h_{3} \alpha=\left[h_{3}, y_{1}\right] \alpha=y_{1} \alpha, \\
& h_{4} y_{1} \alpha=\left[h_{4}, y_{1}\right] \alpha+y_{1} h_{4} \alpha=\left[h_{4}, y_{1}\right] \alpha=0, \\
& X_{1} y_{1} \alpha=\left[X_{1}, y_{1}\right] \alpha+y_{1} X_{1} \alpha=\left[X_{1}, y_{1}\right] \alpha=0, \\
& X_{2} y_{1} \alpha=\left[X_{2}, y_{1}\right] \alpha+y_{1} X_{2} \alpha=\left[X_{2}, y_{1}\right] \alpha=0,
\end{aligned}
$$

as required. Also

$$
x_{1} y_{1} \alpha=\left[x_{1}, y_{1}\right] \alpha+y_{1} x_{1} \alpha=\left[x_{1}, y_{1}\right] \alpha=\left(h_{2}-h_{3}\right) \alpha=0 \text {, }
$$

so this defines an invariant operator $(0,0 \mid 0,0) \rightarrow(0,1 \mid-1,0)$, namely the exterior derivative $\Omega^{0} \rightarrow \Omega^{1}$.

Another symbol is described by the weight vector $y_{1}^{2} \alpha$ :

$$
\begin{aligned}
h_{1} y_{1}^{2} \alpha=0=h_{4} y_{1}^{2} \alpha, \quad h_{2} y_{1}^{2} \alpha & =-2 y_{1}^{2} \alpha, \quad h_{3} y_{1}^{2} \alpha=2 y_{1}^{2} \alpha, \\
X_{1} y_{1}^{2} \alpha=0 & =X_{2} y_{1}^{2} \alpha .
\end{aligned}
$$


However,

$$
\begin{aligned}
x_{1} y_{1}^{2} \alpha & =\left[x_{1}, y_{1}\right] y_{1} \alpha+y_{1} x_{1} y_{1} \alpha=\left(h_{2}-h_{3}\right) y_{1} \alpha \\
& =\left[h_{2}, y_{1}\right] \alpha-\left[h_{3}, y_{1}\right] \alpha=-2 y_{1} \alpha,
\end{aligned}
$$

so the symbol $\odot^{2} \Omega^{1} \otimes(0,0 \mid 0,0)=(0,2 \mid-2,0) \oplus(1,1 \mid-1,-1) \rightarrow(0,2 \mid-2,0)$ does not lift to an invariant operator.

Next consider the Verma module $V(0, n \mid-1,-1)$ generated by highest weight vector $\alpha$. The symbol

$$
\Omega^{1} \otimes(0, n \mid-1,-1)=(0, n+1 \mid-2,-1) \oplus(1, n \mid-2,-1) \rightarrow(1, n \mid-2,-1)
$$

is represented by the weight vector $n y_{2} \alpha+y_{1} Y_{1} \alpha$ (this follows from the proof of the Clebsch-Gordan formula for $\mathfrak{g l}(2, \mathbb{C})$; see e.g. [19]). Moreover,

$$
\begin{aligned}
x_{1}\left(n y_{2} \alpha+y_{1} Y_{1} \alpha\right) & =n\left[x_{1}, y_{2}\right] \alpha+\left[x_{1}, y_{1}\right] Y_{1} \alpha+y_{1} x_{1} Y_{1} \alpha \\
& =n Y_{1} \alpha+\left(h_{2}-h_{3}\right) Y_{1} \alpha+y_{1}\left[x_{1}, Y_{1}\right] \alpha \\
& =n Y_{1} \alpha+Y_{1} \alpha-n Y_{1} \alpha-Y_{1} \alpha=0,
\end{aligned}
$$

so this symbol lifts to an invariant operator $(0, n \mid-1,-1) \rightarrow(1, n \mid-2,-1)$. This is the massless field operator of helicity $n / 2$ described in the introduction. In the notation of $[10]$ it is

$$
\nabla_{A}^{A \prime}: \mathcal{O}_{\left(A^{\prime} B^{\prime} \cdots D^{\prime}\right)}[-1] \rightarrow \mathcal{O}_{A\left(B^{\prime} \cdots D^{\prime}\right)}[-1][-1]^{\prime} .
$$

Finally, consider the Verma module $V(0,0 \mid-1,-1)$ and again denote the generating highest weight by $\alpha$. Consider $\gamma \equiv\left(y_{1} y_{4}-y_{2} y_{3}\right) \alpha$. It is readily verified that $h_{1} \gamma=-\gamma, h_{2} \gamma=-\gamma, h_{3} \gamma=2 \gamma, h_{4} \gamma=2 \gamma$ and that $X_{1}, X_{2}$, and $x_{1}$ annihilate $\gamma$. This highest weight vector therefore corresponds to an invariant operator $(0,0 \mid-1,-1) \rightarrow(1,1 \mid-2,-2)$, namely the wave operator (see e.g. $[10,33]$ )

$$
\square: \mathcal{O}[-1] \rightarrow \mathcal{O}[-2][-1]^{\prime} \text {. }
$$

\section{The Use of the Central Character}

Let $Z(\mathfrak{g})$ denote the centre of $\mathfrak{U}(\mathfrak{g})$, i.e.. the elements which commute with all others in $\mathfrak{U}(\mathfrak{g})$. These elements are often called Casimir operators. For any $\mathfrak{U}(\mathfrak{g})$-module $W$, the elements of $Z(\mathfrak{g})$ act as $\mathfrak{U}(\mathfrak{g})$-module endomorphisms. Consequently they take weight vectors into weight vectors of the same weight and also preserve highest weight vectors. Hence if $\alpha \in V(\rho)$ is a highest weight vector generating $V(\rho)$, then $z \alpha=\phi(z) \alpha$ for some $\phi(z) \in \mathbb{C}$. This defines $\phi$ as an algebra homomorphism $Z(\mathfrak{g}) \rightarrow \mathbb{C}$. Now for any $x \in \mathfrak{U}(\mathfrak{g})$,

$$
z(x \alpha)=x(z \alpha)=\phi(z) x \alpha,
$$

so that $z$ acts on $V(\rho)$ as multiplication by $\phi(z)$. This homomorphism $\phi$ is called the central character or infinitesimal character of $V(\rho)$.

Suppose $D: V(\sigma) \rightarrow V(\rho)$ is a $\mathfrak{U}(\mathfrak{g})$-module homomorphism between Verma modules. If $V(\sigma)$ has central character $\phi$, then for any $v \in V(\sigma)$ and $z \in Z(\mathfrak{g})$,

$$
z D(v)=D(z v)=D(\phi(z) v)=\phi(z) D(v),
$$


so $\phi$ is also the central character of $V(\rho)$ if $D$ is to be non-zero. Hence, in searching for $\mathfrak{U}(\mathfrak{g})$-homomorphisms between Verma modules, one is constrained by the (very strong) condition that the two modules have the same central character.

As an illustration one can use this information to determine all invariant differential operators on the Riemann sphere as a homogeneous space

$$
G L(2, \mathbb{C}) /\left(\begin{array}{ll}
* & * \\
0 & *
\end{array}\right) .
$$

Label the generators of $\mathfrak{g l}(2, \mathbb{C})$ according to

$$
\left(\begin{array}{ll}
h_{1} & x \\
y & h_{2}
\end{array}\right) .
$$

Then $h_{1}+h_{2}$ and $\left(h_{1}-h_{2}\right)^{2}+4 y x-2\left(h_{1}-h_{2}\right)$ lie in (and in fact generate) the centre of $\mathfrak{U}(\mathfrak{g l}(2, \mathbb{C}))$. The irreducible homogeneous bundles are all line bundles. Explicitly, $(a \mid b)$ for any integers $a$ and $b$ corresponds to the representation on $\mathbb{C}$ given by

$$
\left(\begin{array}{ll}
\zeta & * \\
0 & \xi
\end{array}\right) \mapsto \zeta^{a} \xi^{b}
$$

and $V(a \mid b)$ is generated by a highest weight vector $\alpha$ with $h_{1} \alpha=-a \alpha$ and $h_{2} \alpha=$ $-b \alpha$. Hence the two Casimir operators act by

$$
\begin{aligned}
{\left[h_{1}+h_{2}\right] \alpha } & =-[a+b] \alpha, \\
{\left[\left(h_{1}-h_{2}\right)^{2}+4 y x-2\left(h_{1}-h_{2}\right)\right] \alpha } & =\left[(b-a)^{2}+2(b-a)\right] \alpha .
\end{aligned}
$$

In order that $V(e \mid f)$ and $V(a \mid b)$ have the same central character,

$$
a+b=e+f \text { and }(b-a)^{2}+2(b-a)=(e-f)^{2}+2(e-f),
$$

so either $e=a, f=b$ and the homomorphism is a multiple of the identity, or else $e=b+1$ and $f=a-1$. In this second case there is no non-trivial map unless $a \leqq b$, where $y^{b-a+1} \alpha$ is a highest weight vector in $V(a \mid b)$ providing the required homomorphism. This is unique up to scale and there is an exact sequence,

$$
0 \rightarrow V(b+1 \mid a-1) \rightarrow V(a \mid b) \rightarrow(a, b) \rightarrow 0,
$$

where $(a, b)$ is a finite dimensional irreducible representation of $\mathfrak{g l}(2, \mathbb{C})$. This method of constructing representations (such as $(a, b)$ ) is a standard application of the theory [19] of Verma modules. In terms of invariant differential operators this yields the dual exact sequence

$$
0 \rightarrow(a, b) \rightarrow(a \mid b) \rightarrow(b+1 \mid a-1) \rightarrow 0 .
$$

The differential operator is a conformally invariant power of edth [11] and this constitutes a complete list of such globally invariant operators.

Harish Chandra's theorem $[18,19]$ uses an analysis of this kind to identify both the centre $Z(g)$ and those Verma modules which have the same central character via their highest weights. For $\operatorname{gl}(4, \mathbb{C})$ the result is that $V(a, b \mid c, d)$ and $V(e, f \mid g, h)$ have the same central character if and only if $(e+1, f+2, g+3, h+4)$ is a permutation of $(a+1, b+2, c+3, d+4)$. This immediately cuts down $(e, f \mid g, h)$ to at most six 
possibilities. In general $V(\rho)$ and $V(\sigma)$ have the same central character provided $\rho$ and $\sigma$ are related under the affine action of the Weyl group.

Generally, $(a+1, b+2, c+3, d+4)$ will consist of distinct integers in which case $(a, b \mid c, d)$ is said to be regular and there are precisely six representations with the same central character. Explicitly, one can first apply a permutation as above to suppose that, without loss of generality, $a \leqq b \leqq c \leqq d$. The representations with the same infinitesimal character are then:

$$
\begin{aligned}
(a, b \mid c, d)(a, c+1 \mid b-1, d) & \begin{array}{r}
(a, d+2 \mid b-1, c-1) \\
(b+1, c+1 \mid a-2, d)
\end{array} \\
(b+1, d+2 \mid a-2, c-1) & (b+2, d+2 \mid a-2, b-2) .
\end{aligned}
$$

In the case of a singular character (not regular), the same pattern occurs except that if any of the adjacent slots in $(, 1$, ) are in decreasing order, then this possibility is omitted and some distinct representations in the regular pattern may now coincide, e.g.

$$
(0,0 \mid-1,-1)(0,0 \mid-1,-1){ }_{0}^{0}(1,1 \mid-2,-2)(1,1 \mid-2,-2)
$$

or

$$
0(1,1 \mid-1,0) \underset{(1,1 \mid-1,0)}{(1,2 \mid-1,-1)}(1,2 \mid-1,-1) 0 .
$$

It is now possible systematically to determine all invariant differential operators. For example, consider the case

$$
(0,0 \mid 0,0)(0,1 \mid-1,0) \begin{gathered}
(0,2 \mid-1,-1) \\
(1,1 \mid-2,0)
\end{gathered}(1,2 \mid-2,-1)(2,2 \mid-2,-2) .
$$

There are already a limited number of possible symbols. Since

$$
\begin{aligned}
\Omega^{1} & =(0,1 \mid-1,0), \\
\odot^{2} \Omega^{1} & =(0,2 \mid-2,0) \oplus(1,1 \mid-1,-1), \\
\odot^{3} \Omega^{1} & =(0,3 \mid-3,0) \oplus(1,2 \mid-2,-1), \\
\odot^{4} \Omega^{1} & =(0,4 \mid-4,0) \oplus(1,3 \mid-3,-1) \oplus(2,2 \mid-2,-2),
\end{aligned}
$$

there are, for example, only three possible symbols for a differential operator acting on $(0,0 \mid 0,0)$ :

$$
\begin{gathered}
\Omega^{1} \otimes(0,0 \mid 0,0) \rightarrow(0,1 \mid-1,0) \\
\odot^{3} \Omega^{1} \otimes(0,0 \mid 0,0) \rightarrow(1,2 \mid-2,-1) \\
\odot{ }^{4} \Omega^{1} \otimes(0,0 \mid 0,0) \rightarrow(2,2 \mid-2,-2) .
\end{gathered}
$$

The corresponding weight vectors in $V(0,0 \mid 0,0)$ are $y_{1} \alpha, y_{1}\left(y_{1} y_{4}-y_{2} y_{3}\right) \alpha$, and $\left(y_{1} y_{4}-y_{2} y_{3}\right)^{2} \alpha$. The case $y_{1} \alpha$ was investigated at the end of the previous section and yields the exterior derivative,

$$
d: \Omega^{0}=(0,0 \mid 0,0) \rightarrow(0,1 \mid-1,0)=\Omega^{1},
$$


as an invariant operator. A straightforward computation, however, shows that

$$
x_{1}\left(y_{1}\left(y_{1} y_{4}-y_{2} y_{3}\right) \alpha\right)=2 y_{2} y_{3} \alpha-y_{1} y_{4} \alpha \text {, }
$$

so there is no invariant operator $(0,0 \mid 0,0) \rightarrow(1,2 \mid-2,-1)$. Finally, $x_{1}\left(y_{1} y_{4}\right.$ $\left.-y_{2} y_{3}\right)^{2} \alpha=0$, so that there is an invariant operator

$$
\Omega^{0}=(0,0 \mid 0,0) \rightarrow(2,2 \mid-2,-2)=\Omega^{4} .
$$

This is perhaps slightly unexpected. The curved generalization is described in [15].

Continuing in this manner, it is easy to determine all the invariant operators for this basic case to obtain the diagram (not commutative)

$$
\left.(0,0 \mid 0,0) \rightarrow(0,1 \mid-1,0) \bigcup_{(}^{(0,2 \mid-1,-1) \backslash(1,2 \mid}-2,-1\right) \rightarrow(2,2 \mid-2,-2) .
$$

The operator $(0,1 \mid-1,0) \rightarrow(1,2 \mid-2,-1)$ is either of the obvious compositions, each operator is determined up to scale, and, apart from $\Omega^{0} \rightarrow \Omega^{4}$, this is nothing more than the deRham resolution:

$$
0 \rightarrow \mathbb{C} \rightarrow \Omega^{0} \rightarrow \Omega^{1} \underset{\Omega_{-}^{2}}{\Omega_{+}^{2}} \rightarrow \Omega^{3} \rightarrow \Omega^{4} \rightarrow 0
$$

where $\Omega_{+}^{2}$ (respectively $\Omega_{-}^{2}$ ) denotes the (sheaf of germs of) self-dual (respectively anti-self-dual) 2-forms. Of course, this is conformally invariant in the curved case too. On the level of Verma modules, the deRham sequence is reflected in a Koszul complex on $\mathbb{C}\left[y_{1}, y_{2}, y_{3}, y_{4}\right]$.

\section{The Translation Principle}

Although it is possible to treat the general case in a similar manner to the case of $(0,0 \mid 0,0)$ as above, there is a short cut by way of the Jantzen-Zuckerman translation functor (see e.g. [32]). This translation principle is a method for producing new homomorphisms from old ones between Verma modules. The general idea is as follows. Start with $D: V(\sigma) \rightarrow V(\rho)$ for irreducible representations $\rho$ and $\sigma$ of $\mathfrak{p}$. Suppose $\tau: \mathfrak{g} \rightarrow$ End $(W)$ is an irreducible representation and consider

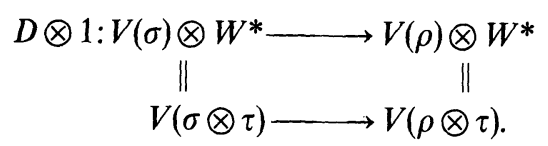

If $\rho \otimes \tau$ and $\sigma \otimes \tau$ contained irreducible representations $\zeta$ and $\eta$ of $\mathfrak{p}$ as direct summands, then the corresponding Verma modules would split off $V(\sigma \otimes \tau)$ and $V(\rho \otimes \tau)$, whence $D \otimes 1$ would give rise to a new homomorphism $V(\eta) \rightarrow V(\zeta)$. Unfortunately, things are rarely so simple since $\sigma \otimes \tau$ is typically reducible but not decomposable. Nevertheless, the corresponding Verma module $V(\sigma \otimes \tau)$ will often split as a genuine direct sum of Verma submodules. The point is that $V(\sigma \otimes \tau)$ is composed of weight spaces which may then be grouped according to central character. Since central character is preserved under the action of $\mathfrak{g}$, it is automatic that this grouping provides a $\mathfrak{U}(\mathfrak{g})$-module splitting of $V(\sigma \otimes \tau)$. Thus, if distinct composition factors of $\sigma \otimes \tau$ have distinct central character, then the corresponding Verma modules occur as direct summands of $V(\sigma \otimes \tau)$. Such a condition is easily checked by Harish Chandra's theorem. If this also happens for $V(\rho \otimes \tau)$, then $D \otimes 1$ 
induces new homomorphisms between these Verma modules. Indeed, since, by earlier reasoning, a homomorphism of Verma modules for irreducible representations of $\mathfrak{p}$ must preserve central character, $D \otimes 1$ must split up so as to act between corresponding direct summands.

Dually, the story is as follows. $D$ is an invariant differential operator between homogeneous vector bundles $E$ and $F$ over $G / P$. Thus, $D$ induces an invariant differential operator between $E \otimes W$ and $F \otimes W$, where $W$ is a representation of $G$ regarded as the trivial bundle with fibre $W$ over $G / P$. Generally, $E \otimes W$ and $F \otimes W$ will not decompose into irreducible homogeneous bundles but irreducible factors may be split off by invariant differential operators, thus yielding new invariant operators when combined with $D$.

To see how this works in practice, consider, for example, the Verma module $V(0,0 \mid 0,0) \otimes(0,0,0,1)^{*}=V(0,0,0,1)$. The representation $(0,0,0,1)$ of $G L(4, \mathbb{C})$ is reducible but not decomposable as a representation of $P$. There is an exact sequence of $P$-representations,

$$
0 \rightarrow(0,1 \mid 0,0) \rightarrow(0,0,0,1) \rightarrow(0,0 \mid 0,1) \rightarrow 0,
$$

which does not split. In the notation of [10] there is an exact sequence of bundles

$$
0 \rightarrow \mathcal{O}_{A^{\prime}} \rightarrow \mathcal{O}^{\alpha} \rightarrow \mathcal{O}^{A} \rightarrow 0
$$

which does not split. But $V(0,1 \mid 0,0)$ and $V(0,0 \mid 0,1)$ have different central character, and so the exact sequence of Verma modules

$$
0 \rightarrow V(0,0 \mid 0,1) \rightarrow V(0,0,0,1) \rightarrow V(0,1 \mid 0,0) \rightarrow 0
$$

canonically splits. For the correspodning homogeneous vector bundles, this implies a splitting

$$
0 \rightarrow(0,1|\underbrace{0,0) \rightarrow(0,0,0}_{D}, \underbrace{, 1) \rightarrow(0,0}_{D}| 0,1) \rightarrow 0,
$$

where $D$ and $\widetilde{D}$ are (first order) differential operators. In other words, although this sequence does not split as vector bundles, as a sequence of sheaves it does split. We shall denote this phenomenon with the notation (cf. [13])

$$
(0,0,0,1)=(0,0 \mid 0,1)+(0,1 \mid 0,0) .
$$

Explicit formulae for $D$ and $\widetilde{D}$ will be given in the next section. We claim that, more generally there is a series of splittings

$$
0 \rightarrow(a, b \mid c, d) \underbrace{\otimes(0,1 \mid 0,0) \rightarrow(a, b \mid c, d)}_{D} \otimes \underbrace{(0,0,0,1) \rightarrow(a, b \mid c, d) \otimes}_{\tilde{D}}(0,0 \mid 0,1) \rightarrow 0
$$

by invariant differential operators $D$ and $\tilde{D}$ provided $(a, b \mid c, d)$ is regular. To see this first observe that, by the Clebsch-Gordan formula (e.g. [19]),

$$
\begin{aligned}
& (a, b \mid c, d) \otimes(0,1 \mid 0,0)=(a, b+1 \mid c, d) \oplus(a+1, b \mid c, d) \\
& \quad \text { (omitted if } a=b), \\
& (a, b \mid c, d) \otimes(0,0 \mid 0,1)=(a, b \mid c, d+1) \oplus(a, b \mid c+1, d)
\end{aligned}
$$

(omitted if $c=d$ ). 
These will all have distinct central character provided $\{a+1, b+3, c+3, d+4\}$, $\{a+2, b+2, c+3, d+4\},\{a+1, b+2, c+4, d+4\}$, and $\{a+1, b+2, c+3, d+5\}$ are distinct. This is indeed the case provided $(a, b \mid c, d)$ is regular, i.e. provided $a+1, b+2, c+3$, and $d+4$ are distinct. Thus $D$ and $\widetilde{D}$ exist by earlier general reasoning. Again, explicit formulae will be given in the next section. By symbol considerations, $D$ and $\tilde{D}$ are first order.

To see the translation principle in action, consider the invariant differential operator $d: \Omega^{0}=(0,0 \mid 0,0) \rightarrow(0,1 \mid-1,0)=\Omega^{1}$ and tensor through by $(0,0,0,1)$. This gives

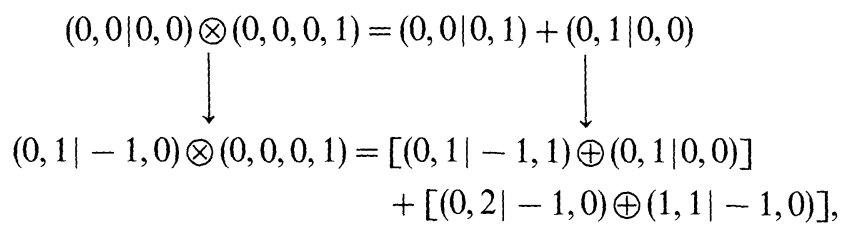

and hence a new invariant differential operator

$$
(0,0 \mid 0,1) \rightarrow(0,1 \mid-1,1)
$$

together with the identity $(0,1 \mid 0,0) \rightarrow(0,1 \mid 0,0)$. Recall that $(0,0 \mid 0,0) \rightarrow(0,1 \mid-1,0)$ had $\mathbb{C}$ as kernel, so that

$$
0 \rightarrow \mathbb{C}(0,0,0,1) \rightarrow(0,0,0,1) \rightarrow(0,1 \mid-1,0) \otimes(0,0,0,1)
$$

is exact, where $\mathbb{C}(0,0,0,1)$ denotes the finite dimensional representation of $G L(4, \mathbb{C})$ as distinct from the corresponding homogeneous bundle. This implies that $\mathbb{C}(0,0,0,1)$ is also the kernel of the new operator $(0,0 \mid 0,1) \rightarrow(0,1 \mid-1,1)$. Applying this process to the whole deRham sequence yields an invariant resolution

$$
\begin{aligned}
0 \rightarrow & \mathbb{C}(0,0,0,1) \rightarrow(0,0 \mid 0,1) \\
& \rightarrow(0,1 \mid-1,1) \underset{\searrow}{(0,3 \mid-1,-1)}(1,1 \mid-2,1) \rightarrow(1,3 \mid-2,-1) \rightarrow(2,3 \mid-2,-2) \rightarrow 0
\end{aligned}
$$

of $(0,0,0,1)$ and an invariant (fifth order) differential operator

$$
(0,0 \mid 0,1) \rightarrow(2,3 \mid-2,-2)
$$

obtained from $(0,0 \mid 0,0) \rightarrow(2,2 \mid-2,-2)$. One can recover the original case by tensoring this new case through by $(-1,0,0,0)$ and using

$$
\begin{aligned}
& (-1,0,0,0) \otimes(0,0,0,1)=(-1,0,0,1) \oplus(0,0,0,0) \text { and } \\
& (a, b \mid c, d) \otimes(-1,0,0,0)=(a, b \mid c, d) \otimes(-1,0 \mid 0,0)+(a, b \mid c, d) \otimes(0,0 \mid-1,0)
\end{aligned}
$$

for regular $(a, b \mid c, d)$. This also shows that there are no extra invariant operators (up to scale) between the bundles

$$
(0,0 \mid 0,1) \rightarrow(0,1 \mid-1,1) \rightarrow \begin{gathered}
(0,3 \mid-1,-1) \\
(1,1 \mid-2,1)
\end{gathered} \rightarrow(1,3 \mid-2,-1) \rightarrow(2,3 \mid-2,-2)
$$

other than those shown (else they would give new operators for the $(0,0 \mid 0,0)$ case).

One can continue on this way to investigate all regular homogeneous bundles 
$(a, b \mid c, d)$ by tensoring repeatedly with $(0,0,0,1)$ and $(-1,0,0,0)$. This is the translation principle and yields the following:

Theorem. For $a \leqq b \leqq c \leqq d$ there are non-zero invariant holomorphic differential operators as in the following (non-commutative) diagram

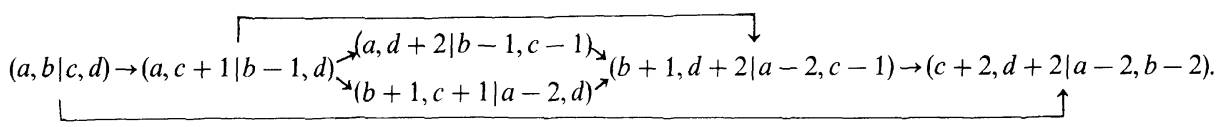

Each operator is unique up to scale, and this is a complete list of invariant operators between regular irreducible homogeneous bundles. Omitting the operator $(a, b \mid c, d) \rightarrow(c+2, d+2 \mid a-2, b-2)$ from the above diagram leaves a resolution of $\mathbb{C}(a, b, c, d)$.

Remarks. The resolution in this theorem may also be deduced from the BernsteinGelfand-Gelfand resolution [3] or constructed by analogous reasoning [21]. In [14] it is referred to as the generalized deRham sequence. The invariant operators between singular homogeneous bundles may be investigated in a fashion analogous to the regular case. The translation principle applies within the realm of singular bundles and results in diagrams degenerating from the regular case as in the following examples:

$$
\begin{aligned}
& \underset{\uparrow}{(0,0) \mid-1,-1) \longrightarrow(0,0 \mid-1,-1)} \begin{array}{l}
0 \\
\uparrow
\end{array} \quad(1,1 \mid-2,-2)=(1,1 \mid-2,-2) \\
& 0 \quad(1,1 \mid-1,0) \longrightarrow(1,2 \mid-1,-1) \longrightarrow(1,2 \mid-1,-1) \quad 0 .
\end{aligned}
$$

Further investigation of these operators is best undertaken in the context of general four-dimensional conformal geometry.

\section{The Curved Case}

The link between the homogeneous approach and the general four-dimensional Riemannian manifold $M$ is via the diagram of Lie groups (see e.g. [27])

$$
\begin{gathered}
S L(2, \mathbb{C}) \times S L(2, \mathbb{C}) \rightarrow S O(4, \mathbb{C}) \text { a } 2-1 \text { covering, } \\
\cap \\
S(G L(2, \mathbb{C}) \times G L(2, \mathbb{C})) \rightarrow C O(4, \mathbb{C}) \quad a 4-1 \text { covering, }
\end{gathered}
$$

where $C O(4, \mathbb{C})$ is the complex conformal group. We shall assume that $M$ has only a conformal metric, i.e. a reduction of the structure group of the tangent bundle from $G L(4, \mathbb{C})$ to $C O(4, \mathbb{C})$ and that, moreover, $M$ is spin, i.e. there is a lifting of this $C O(4, \mathbb{C})$-bundle to a $S(G L(2, \mathbb{C}) \times G L(2, \mathbb{C}))$-bundle. This is always the case locally, and then some of the conformally invariant operators which can be constructed locally will fail globally to exist simply through lack of spinors, whereas those which involve only tensors will be globally well-defined. Hence, this is not a serious restriction. An irreducible spinor bundle is the induced bundle for an irreducible 
representation of $S(G L(2, \mathbb{C}) \times G L(2, \mathbb{C}))$, whereas the irreducible homogeneous bundles on $\mathbb{M}$ earlier in this paper were given by irreducible representations of $L=G L(2, \mathbb{C}) \times G L(2, \mathbb{C})$. This is a rather trivial change, and we shall continue to use the same notation, i.e. $(a, b \mid c, d)$ now denotes an irreducible spinor bundle on $M$. With notation analogous to that in [10],

$$
(a, b \mid c, d)=\mathcal{O}(\overbrace{A B \cdots D}^{d-c})(\overbrace{E^{\prime} F^{\prime} \cdots H^{\prime}}^{b-a}\{d-a\},
$$

meaning that sections of $(a, b \mid c, d)$ are spinor fields with $d-c$ symmetric unprimed indices, $b-a$ symmetric primed indices, and of conformal weight $d-a$ (see [27] for definitions and further discussion (cf. also [10])). In the language of [10], primed and unprimed conformal weights have been identified. Thus,

$$
(a, b \mid c, d)=(a+n, b+b \mid c+n, d+n)
$$

for any integer $n$.

The idea in the curved case is to mimick the construction of Sect. 5 using the methods of, for example, [27] to check conformal invariance at each stage. In the conformally flat case the construction will reduce to that of Sect. 5.

The first thing which is needed is a curved analogue of the basic case

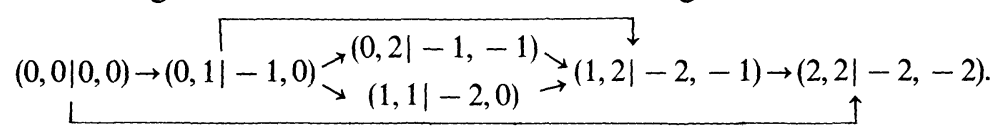

This is nothing more than the deRham sequence together with [15]

$$
(0,0 \mid 0,0)=\Omega^{0} \stackrel{\nabla_{b}\left[\nabla^{b} \nabla^{a}-2 R^{a b}+2 R g^{a b} / 3\right] \nabla_{a}}{\longrightarrow} \Omega^{4}=(2,2 \mid-2,-2),
$$

where $g_{a b}$ is any metric in the conformal class, $\nabla_{a}$ is the metric connection, $R_{a b}$ is the Ricci curvature, and $R$ is the scalar curvature with normalization conventions taken from [27].

To invoke the translation principle in the general case, the next ingredient is a curved version of

$$
0 \rightarrow(0,1 \mid 0,0) \rightarrow(0,0,0,1) \rightarrow(0,0 \mid 0,1) \rightarrow 0 .
$$

For this take $(0,0,0,1)$ to be the bundle of local twistors $[26,28]$ constructed as follows. For any choice of metric $g_{a b}$ the sequence is required to split (as bundles) whereby

$$
(0,0,0,1)=\underset{(0,1 \mid 0,0)}{\bigoplus}=\underset{\mathcal{O}_{A^{\prime}}}{\oplus}\left(\begin{array}{l}
(0,0 \mid 0,1) \\
\mathcal{O}_{A^{\prime}}
\end{array}\right),
$$

but if $g_{a b}$ is replaced by $\hat{g}_{a b}=\Omega^{2} g_{a b}$, then this splitting is changed according to

$$
\hat{\omega}^{A}=\omega^{A}, \quad \hat{\pi}_{A^{\prime}}=\pi_{A^{\prime}}-\Upsilon_{A A^{\prime}} \omega^{A}, \text { where } \Upsilon_{a}=\Omega^{-1} \nabla_{a} \Omega .
$$

The resulting exact sequence is evidently conformally invariant.

In the flat case $(0,0,0,1)$ is a canonically trivial bundle. One way of describing this triviality is as a flat connection. There is a notion $[26,28]$ of local twistor connection (or transport) $D_{a}$ which generalizes this to the curved case. Specifically, for a chosen metric $g_{a b}$ in the conformal class, $D_{a}$ is defined by 


$$
D_{b}\left(\begin{array}{l}
\omega^{A} \\
\pi_{A^{\prime}}
\end{array}\right)=\left(\begin{array}{l}
\nabla_{B B^{\prime}} \omega^{A}+\delta_{B}^{A} \pi_{A^{\prime}} \\
\nabla_{B B^{\prime}} \pi_{A^{\prime}}-P_{A B A^{\prime} B^{\prime}} \omega^{A}
\end{array}\right),
$$

where $\nabla_{a}$ is the metric connection, $\delta_{B}^{A}$ is the Kronecker delta, and $P_{a b}=-R_{a b} / 2$ $+R g_{a b} / 12$. Using formulae from [27], this definition is easily seen to be conformally invariant as follows:

$$
\begin{aligned}
& \begin{aligned}
& \hat{D}_{b}\left(\begin{array}{l}
\omega^{A} \\
\pi_{A^{\prime}}
\end{array}\right)=\left(\begin{array}{l}
\hat{\nabla}_{B B^{\prime}} \hat{\omega}^{A}+\delta_{B}^{A} \hat{\pi}_{B^{\prime}} \\
\hat{\nabla}_{B B^{\prime}} \hat{\pi}_{A^{\prime}}-\hat{P}_{A B A^{\prime} B^{\prime}} \hat{\omega}^{A}
\end{array}\right)=\left(\begin{array}{l}
\hat{\nabla}_{B B^{\prime}} \omega^{A}+\delta_{B}^{A}\left(\pi_{B^{\prime}}-\Upsilon_{C B^{\prime}} \omega^{C}\right) \\
\hat{\nabla}_{B B^{\prime}}\left(\pi_{A^{\prime}}-\Upsilon_{A A^{\prime}} \omega^{A}\right)-\hat{P}_{A B A^{\prime} B^{\prime}} \omega^{A}
\end{array}\right) \\
&=\left(\begin{array}{l}
\nabla_{B B^{\prime}} \omega^{A}+\delta_{B}^{A} \Upsilon_{C B^{\prime}} \omega^{C}+\delta_{B}^{A} \pi_{B^{\prime}}-\delta_{B}^{A} \Upsilon_{C B^{\prime}} \omega^{C} \\
\nabla_{B B^{\prime}} \pi_{A^{\prime}}-\left(\nabla_{B B^{\prime}} \Upsilon_{A A^{\prime}}\right) \omega^{A}-\Upsilon_{A A^{\prime}} \nabla_{B B^{\prime}} \omega^{A}-\Upsilon_{B A^{\prime}}\left(\pi_{B^{\prime}}-\Upsilon_{A B^{\prime}} \omega^{A}\right)-\hat{P}_{A B A^{\prime} B^{\prime}} \omega^{A}
\end{array}\right), \\
& \text { since } \hat{P}_{A B A^{\prime} B^{\prime}}=P_{A B A^{\prime} B^{\prime}}-\nabla_{B B^{\prime}} \Upsilon_{A A^{\prime}}+\Upsilon_{B A^{\prime}} \Upsilon_{A B^{\prime}}=\left(\begin{array}{l}
\nabla_{B B^{\prime}} \omega^{A}+\delta_{B}^{A} \pi_{B^{\prime}} \\
\nabla_{B B^{\prime}} \pi_{A^{\prime}}-P_{A B A^{\prime} B^{\prime}} \omega^{A}
\end{array}\right),
\end{aligned}
\end{aligned}
$$

as required.

The final ingredient is a series of splittings

$$
0 \rightarrow(a, b \mid c, d) \otimes_{D} \underbrace{(0,1 \mid 0,0) \rightarrow(a, b \mid c, d)}_{\tilde{D}} \otimes \underbrace{(0,0 \mid 0,1) \rightarrow 0}_{(0,0,0,1) \rightarrow(a, b \mid c, d)}
$$

by conformally invariant first order differential operators $D$ and $\widetilde{D}$. It is straightforward though tedious to check that $D$ may be defined by

$$
\begin{aligned}
D\left(\begin{array}{c}
\omega_{B \cdots D E^{\prime} \cdots G^{\prime}}^{A} \\
\pi_{A^{\prime} B \cdots D E^{\prime} \cdots G^{\prime}}
\end{array}\right)= & \pi_{A^{\prime} E^{\prime} \cdots G^{\prime}}^{B \cdots D}+\frac{1}{d-b+2} \nabla_{A\left(A^{\prime}\right.} \sigma_{\left.E^{\prime} \cdots G^{\prime}\right)}^{A B \cdots D}-\frac{b-a}{(b-a+1)(d-a+3)} \\
& \cdot \nabla_{A}^{\left.H^{\prime} \varepsilon_{A^{\prime}\left(E^{\prime}\right.} \sigma_{\left.F^{\prime} \cdots G^{\prime}\right) H^{\prime}}^{A B \cdots D}-\frac{1}{c-b+1} \nabla_{\left(A^{\prime}\right.}^{(B} \tau_{\left.E^{\prime} \cdots G^{\prime}\right)}^{C \cdots D}\right)} \\
& +\frac{b-a}{(b-a+1)(c-a+2)} \nabla^{H^{\prime}(B} \varepsilon_{A^{\prime}\left(E^{\prime}\right.} \tau_{\left.F^{\prime} \cdots G^{\prime}\right) H^{\prime}}^{C \cdots D)},
\end{aligned}
$$

where $\omega_{A B \cdots D E^{\prime} \cdots G^{\prime}}=\sigma_{A B \cdots D E^{\prime} \cdots G^{\prime}}+\varepsilon_{A(B} \tau_{C \cdots D) E^{\prime} \cdots G^{\prime}}$ for $\sigma_{A B \cdots D E^{\prime} \cdots G^{\prime}}=\sigma_{(A B \cdots D)\left(E^{\prime} \cdots G^{\prime}\right)}$ and $\tau_{C \cdots D E^{\prime} \cdots G^{\prime}}=\tau_{(C \cdots D)\left(E^{\prime} \cdots G^{\prime}\right)}$. Here, round brackets on spinor indices denotes symmetrization (see [27]). If $c=d$, then $\tau_{C \cdots D E^{\prime} \cdots G^{\prime}}$ is omitted. Note that the above formula fails if $b=d+2, a=d+3, b=c+1$, or $a=c+2$, i.e. when the character $(a, b \mid c, d)$ is singular. The rest of this section will discuss the curved translation principle only amongst regular characters (for which the above formula is valid). There is a degenerate version which allows one to translate between singular characters exactly as in the flat case.

The idea is now to use the above ingredients in order to generate progressively more complicated invariant operators just as one would in the conformally flat, i.e. homogeneous, case. The crucial observation (made, for example in [27]) is that any conformally invariant formula involving algebraic combinations of the Levi Civita connection and curvature correction terms (as above) remains invariant if the fields also have values in some vector bundle with connection, where the Levi Civita connection is replaced by the background-coupled connection. This observation is used repeatedly with the local twistor bundle $(0,0,0,1)$. 
The curved translation principle works as follows. Start with the basic case

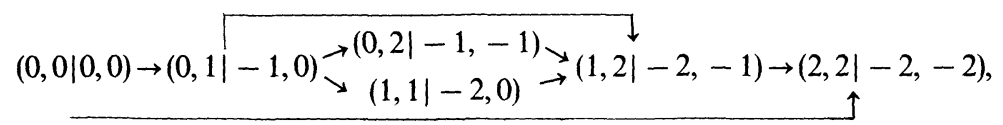

and couple this with $(0,0,0,1)$ to obtain, for example

$$
(0,0,0,1) \stackrel{D_{a}}{\longrightarrow}(0,1 \mid-1,0) \otimes(0,0,0,1) \longrightarrow(0,2 \mid-1,-1) \otimes(0,0,0,1),
$$

given explicitly by

$$
\left(\begin{array}{l}
\omega^{A} \\
\pi_{A^{\prime}}
\end{array}\right) \mapsto\left(\begin{array}{l}
\nabla_{B B^{\prime}} \omega^{A}+\delta_{B}^{A} \pi_{B^{\prime}} \\
\nabla_{B B^{\prime}} \pi_{A^{\prime}}-P_{A B A^{\prime} B^{\prime}} \omega^{A}
\end{array}\right), \quad\left(\begin{array}{l}
\omega_{b}^{A} \\
\pi_{A^{\prime} b}
\end{array}\right) \mapsto\left(\begin{array}{l}
\nabla_{\left(B^{\prime}\right.}^{B} \omega_{\left.C^{\prime}\right) B}^{A}-\pi_{\left(B^{\prime} C^{\prime}\right) A} \\
\nabla_{\left(B^{\prime}\right.}^{B} \pi_{\left.\left|A^{\prime}\right| C\right) B}+\omega_{\left(B^{\prime}\right.}^{A B} P_{\left.C^{\prime}\right) A^{\prime} B A}
\end{array}\right),
$$

where vertical lines round an index indicate that it does not enter the symmetrization. The first of these gives

$$
\begin{aligned}
(0,0,0,1)= & (0,0 \mid 0,1)+(0,1, \mid 0,0) \\
\downarrow & \downarrow \\
(0,1 \mid-1,0) \otimes(0,0,0,1)= & {[(0,1 \mid-1,1) \oplus(0,1 \mid 0,0)] } \\
& +[(0,2 \mid-1,0) \oplus(1,1 \mid-1,0)],
\end{aligned}
$$

and hence a total of eight possible new conformally invariant differential operators. Consider, for example, the composition

$$
\begin{aligned}
& \omega^{A} \stackrel{\tilde{D}}{\longmapsto}\left(\begin{array}{c}
\omega^{A} \\
-\nabla_{A A^{\prime}} \omega^{A} / 2
\end{array}\right) \mapsto\left(\begin{array}{l}
\nabla_{B B^{\prime}} \omega^{A}-\delta_{B}^{A} \nabla_{C A^{\prime}} \omega^{C} / 2 \\
-\nabla_{B B^{\prime}} \nabla_{A A^{\prime}} \omega^{A} / 2-P_{A B A^{\prime} B^{\prime}} \omega^{A}
\end{array}\right) \\
& \pi \quad \pi \quad \pi \\
& (0,0 \mid 0,1) \longrightarrow(0,0,0,1) \longrightarrow(0,1 \mid 1,0) \otimes(0,0,0,1) \rightarrow(0,1 \underset{\Psi}{\mid}-1,1) \oplus(0, \underset{w}{\mid} 0,0) \\
& \left(\begin{array}{c}
\omega_{b}^{A} \\
\pi_{A^{\prime} b}
\end{array}\right) \longmapsto \omega_{B^{\prime}}^{(A B)}+\omega_{A B^{\prime}}^{A}
\end{aligned}
$$

This gives invariant operators

$$
\begin{aligned}
& (0,0 \mid 0,1) \rightarrow(0,1 \mid-1,1) \quad \text { and } \quad(0,0 \mid 0,1) \rightarrow(0,1 \mid 0,0)
\end{aligned}
$$

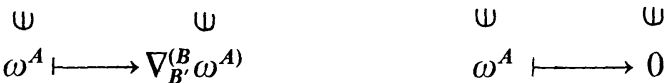

Similar compositions

$$
\begin{aligned}
& \left(\begin{array}{c}
\omega_{b}^{A} \\
\pi_{A^{\prime} b^{\prime}}^{A}
\end{array}\right) \mapsto \pi_{\left(A^{\prime} B^{\prime}\right)}^{B}+\nabla_{A\left(A^{\prime}\right.} \omega_{\left.B^{\prime}\right)}^{(A B)}+\nabla_{\left(A^{\prime}\right.}^{B} \omega_{\left.B^{\prime}\right)}{ }^{A} / 2 \\
& (0,0 \mid 0,1) \rightarrow(0,0,0,1) \rightarrow(0,1 \mid-1,0) \underset{\Psi}{\otimes}(0,0,0,1) \rightarrow(0,2 \mid-1,0) \oplus(1,1 \mid-1,0) \\
& \left(\begin{array}{l}
\omega_{b}^{A} \\
\pi_{A^{\prime} b^{\prime}}
\end{array}\right) \mapsto \pi_{A^{\prime}}^{B A^{\prime}}-\nabla_{A}^{H^{\prime}} \omega_{H^{\prime}}^{(A B)} / 3+\nabla^{H^{\prime} B} \omega_{H^{\prime} A}^{A} / 2
\end{aligned}
$$

give only zero operators:

$$
\begin{aligned}
\omega^{A} & \mapsto-\nabla_{B\left(B^{\prime}\right.} \nabla_{\left.A^{\prime}\right) A} \omega^{A} / 2-P_{A B\left(A^{\prime} B^{\prime}\right)} \omega^{A}+\nabla_{A\left(A^{\prime}\right.} \nabla_{\left.B^{\prime}\right)}^{(A} \omega^{B)} \\
& =-\Phi_{A B A^{\prime} B^{\prime}} \omega^{A}+\left[\nabla_{A\left(A^{\prime}\right.}, \nabla_{\left.B^{\prime}\right) B}-\nabla_{B\left(A^{\prime}\right.} \nabla_{\left.B^{\prime}\right) A}\right] \omega^{A} / 2+\nabla_{A\left(A^{\prime}\right.} \nabla_{\left.B^{\prime}\right)}^{A} \omega_{B} / 2
\end{aligned}
$$




$$
\begin{aligned}
& =-\Phi_{A B A^{\prime} B^{\prime}} \omega^{A}+\varepsilon_{A B} \nabla_{C\left(A^{\prime}\right.} \nabla_{\left.B^{\prime}\right)}^{C} \omega^{A} / 2+\nabla_{A\left(A^{\prime}\right.} \nabla_{\left.B^{\prime}\right)}^{A} \omega_{B} / 2 \\
& =-\Phi_{A B A^{\prime} B^{\prime}} \omega^{A}+\nabla_{A\left(A^{\prime}\right.} \nabla_{\left.B^{\prime}\right)}^{A} \omega_{B}=0, \\
\omega^{A} & \mapsto-\nabla_{B}^{A^{\prime} \nabla_{A A^{\prime}}} \omega^{A} / 2-\left[\nabla_{A}^{H^{\prime}}\left[\nabla_{B H^{\prime}} \omega^{A}-\delta_{B}^{A} \nabla_{C H^{\prime}} \omega^{C} / 2\right]\right] / 3-P_{A B A^{\prime}}{ }^{\prime} \omega^{A} \\
& =-\nabla_{B}^{A^{\prime}} \nabla_{A A^{\prime}} \omega^{A} / 2-\nabla_{A}^{H^{\prime}} \nabla_{B H^{\prime}} \omega^{A} / 3+\nabla_{B}^{H^{\prime}} \nabla_{A H^{\prime}} \omega^{A} / 6+2 \varepsilon_{A B} \Lambda \omega^{A} \\
& =-2 \nabla_{(A}^{A^{\prime}} \nabla_{B) A^{\prime}} \omega^{A} / 3+2 \Lambda \omega_{B}=0,
\end{aligned}
$$

where notation and formulae have been freely taken from [27].

Since the central character is unavailable in the curved case, it is conceivable that, by translation, one can generate more operators than in the flat case. Preliminary calculations such as those above indicate that this does not happen, but we have no proof as yet. The operators which parallel the homogeneous ones have, by construction, the same symbol as in the homogeneous case and differ only by curvature "correction" terms. A more interesting example of such an operator is given by the following composition

$$
\phi_{A B B^{\prime}} \longmapsto\left(\begin{array}{l}
\phi_{b}^{A} \\
2 \nabla_{A^{\prime}}^{A} \phi_{A B B^{\prime}} / 3+\nabla_{B^{\prime}}^{A} \phi_{A B A^{\prime}} / 3
\end{array}\right) \quad\left(\begin{array}{l}
\omega_{B^{\prime} C^{\prime}}^{A} \\
\pi_{A^{\prime} B^{\prime} C^{\prime}}
\end{array}\right) \mapsto \pi_{\left(A^{\prime} B^{\prime} C^{\prime}\right)}-\nabla_{A\left(A^{\prime}\right.} \omega_{\left.B^{\prime} C^{\prime}\right)}^{A}
$$

m

m

m

m

$(0,1 \mid-1,1) \stackrel{\tilde{D}}{\longrightarrow}(0,1 \mid-1,0) \otimes(0,0,0,1) \rightarrow(0,2 \mid-1,-1) \otimes(0,0,0,1) \stackrel{D}{\longrightarrow}(0,3 \mid-1,-1)$

$$
\left(\begin{array}{c}
\omega_{b}^{A} \\
\pi_{A^{\prime} b}
\end{array}\right) \longmapsto\left(\begin{array}{c}
\underset{\nabla^{\prime}}{B} \omega_{\left(B^{\prime}\right.}^{A} \omega_{\left.C^{\prime}\right) B}-\pi_{\left(B^{\prime} C^{\prime}\right)}^{A} \\
\nabla_{\left(B^{\prime}\right.}^{B} \pi_{\left.\left|A^{\prime}\right| C^{\prime}\right) B}+\omega_{\left(B^{\prime}\right.}^{A B} P_{\left.C^{\prime}\right) A^{\prime} B A}
\end{array}\right)
$$

which gives

$$
\begin{aligned}
\phi_{A B B^{\prime}} & \mapsto\left(\begin{array}{l}
\nabla_{\left(B^{\prime}\right.}^{B} \phi_{\left.C^{\prime}\right) B}^{A}-\nabla_{\left(B^{\prime}\right.}^{B} \phi_{\left.C^{\prime}\right) B}^{A} \\
2 \nabla_{\left(B^{\prime}\right.}^{B} \nabla_{\left|A^{\prime}\right|}^{A} \phi_{\left.C^{\prime}\right) A B} / 3+\nabla_{\left(B^{\prime}\right.}^{B} \nabla_{\left.C^{\prime}\right)}^{A} \phi_{A B A^{\prime}} / 3+\phi_{\left(B^{\prime}\right.}^{A B} P_{\left.C^{\prime}\right) A^{\prime} B A}
\end{array}\right) \\
& =\left(\begin{array}{l}
0 \\
\nabla_{\left(A^{\prime}\right.}^{A}, \nabla_{B^{\prime}}^{B} \phi_{\left.C^{\prime}\right) A B}+\Phi_{\left(A^{\prime} B^{\prime}\right.}^{A B} \phi_{\left.C^{\prime}\right) A B}
\end{array}\right) \\
& \mapsto \nabla_{\left(A^{\prime}\right.}^{A} \nabla_{B^{\prime}}^{B} \phi_{\left.C^{\prime}\right) A B}+\Phi_{\left(A^{\prime} B^{\prime}\right.}^{A B} \phi_{\left.C^{\prime}\right) A B} .
\end{aligned}
$$

Although the curved translation principle provides a method of computing exactly what curvature correction terms need be added in general to create a conformally invariant operator, it is, as the above example illustrates, a rather lengthy and inefficient process. In practice it is much easier to start with the leading order term (which must be as in the flat case) and to see how it alters under conformal rescaling in order to ascertain what the lower order terms must be. The translation principle guarantees that this process will generate a consistent answer. Consider for example the operator

$$
(0,1 \mid-1,1)=\mathcal{O}_{(A B) C^{\prime}}\{1\} \rightarrow \mathcal{O}_{\left(A^{\prime} B^{\prime} C^{\prime}\right)}\{-1\}=(0,3 \mid-1,-1) .
$$

From the formulae in [27]:

$$
\hat{\nabla}_{\left(B^{\prime}\right.}^{B} \hat{\phi}_{\left.C^{\prime}\right) A B}=\nabla_{\left(B^{\prime}\right.}^{B} \phi_{\left.C^{\prime}\right) A B}+\Upsilon_{\left(B^{\prime}\right.}^{B} \phi_{\left.C^{\prime}\right) A B},
$$


and thus

$$
\begin{aligned}
\hat{\nabla}_{\left(A^{\prime}\right.}^{A}, \hat{\nabla}_{B^{\prime}}^{B} \hat{\phi}_{\left.C^{\prime}\right) A B}= & \Omega^{-1}\left[\nabla_{\left(A^{\prime}\right.}^{A} \nabla_{B^{\prime}}^{B} \phi_{\left.C^{\prime}\right) A B}+\left[\nabla_{\left(A^{\prime}\right.}^{A}, Y_{B^{\prime}}^{B}\right] \phi_{\left.C^{\prime}\right) A B}\right. \\
& -\Upsilon_{\left(A^{\prime}\right.}^{A} Y_{B^{\prime}}^{B} \phi_{\left.C^{\prime}\right) A B} .
\end{aligned}
$$

But

$$
\Phi_{A^{\prime} B^{\prime}}^{A B}=\Omega^{-2}\left[\Phi_{A^{\prime} B^{\prime}}^{A B}-\nabla_{A^{\prime}}^{(A} \Upsilon_{B^{\prime}}^{B)}+\Upsilon_{A^{\prime}}^{(A} \Upsilon_{B^{\prime}}^{B)}\right], \text { so } \nabla_{\left(A^{\prime}\right.}^{A} \nabla_{B^{\prime}}^{B} \phi_{\left.C^{\prime}\right) A B}+\Phi_{\left(A^{\prime} B^{\prime}\right.}^{A B} \phi_{\left.C^{\prime}\right) A B}
$$

is conformally invariant as obtained above.

In any case one obtains the following diagram of conformally invariant operators

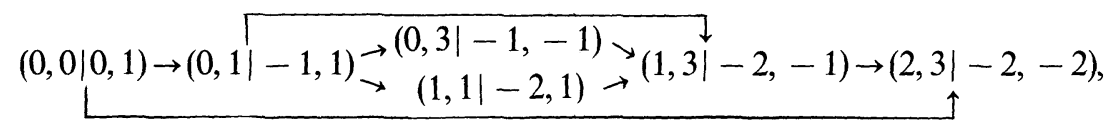

but now omitting the operator $(0,0 \mid 0,1) \rightarrow(2,3 \mid-2,-2)$ no longer leaves an exact sequence. Indeed, this is no longer a complex because the deR ham sequence coupled to the local twistor connection gives the curvature of this connection when two operators are composed. The curvature of the local twistor connection is [28] precisely the Weyl curvature. The composition

$$
(0,0 \mid 0,1) \rightarrow(0,1 \mid-1,1) \rightarrow(1,1 \mid-2,1)
$$

is given by $\psi^{A} \mapsto \Psi_{A B C D} \psi^{A}$, where $\Psi_{A B C D} \in \Gamma(M,(1,1 \mid-3,1))$ is the anti-self-dual Weyl curvature.

Coupling the previous diagram with local twistors and splitting it up using $D$ and $\tilde{D}$ gives two new diagrams:

$$
\begin{aligned}
& (0,0 \mid 0,2) \rightarrow(0,1 \mid-1,2)>(0,4 \mid-1,-1)>(1,4 \mid-2,-1) \rightarrow(2,4 \mid-1,-1) \\
& (0,0 \mid 1,1) \rightarrow(0,2 \mid-1,1) \rightarrow(0,3 \mid-1,0) \nwarrow(1,3 \mid-2,0) \rightarrow(3,3 \mid-2,-2) .
\end{aligned}
$$

For example, $(0,0 \mid 1,1) \rightarrow(0,2 \mid-1,1)$ is given by composing

$$
\omega \longmapsto\left(\begin{array}{l}
\delta_{B}^{A} \omega \\
-\nabla_{A^{\prime} B} \omega
\end{array}\right)
$$

m

$m$

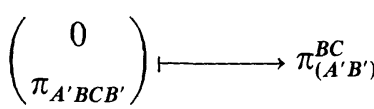

$m$

$$
(0,0 \mid 1,1) \stackrel{\bar{D}}{\longrightarrow}(0,0 \mid 0,1) \otimes(0,0,0,1) \rightarrow(0,1 \mid-1,1) \otimes(0,0,0,1) \stackrel{D}{\longrightarrow}(0,2 \mid-1,1)
$$

$$
\left(\begin{array}{l}
\stackrel{\Psi}{\omega_{B}^{A}} \\
\pi_{A^{\prime} B^{\prime}}
\end{array}\right) \longmapsto\left(\begin{array}{l}
\nabla_{B^{\prime}(B} \omega_{C)}^{A} \stackrel{\Psi}{\Psi} \delta_{(B}^{A} \pi_{C) B^{\prime}} \\
\nabla_{B^{\prime}(B} \pi_{C) A^{\prime}}-P_{A^{\prime} B^{\prime} A(B} \omega_{C)}^{A}
\end{array}\right)
$$

to give

$$
\left[\nabla_{\left(A^{\prime}\right.}^{(A}, \nabla_{\left.B^{\prime}\right)}^{B)}+\Phi_{A^{\prime} B^{\prime}}^{A B}\right] \omega,
$$

though it is easier to work out this correction term $\Phi_{A^{\prime} B^{\prime}}^{A B}$ by inspection, as in previous cases. 
By construction, the anti-self-dual Weyl curvature

$$
\Psi_{A B C D} \in \Gamma(M,(1,1 \mid-3,1))
$$

is conformally invariant. This spinor field may itself be the subject of an invariant operator:

$$
\begin{aligned}
& (1,1 \mid-3,1)-(1,3 \mid-3,-1) \\
& \Psi_{A B C D}^{U} \longmapsto \mathbb{U}_{a b}^{\Psi} .
\end{aligned}
$$

As remarked by Roger Penrose, this gives the Bach tensor (e.g. [16,28]), a wellknown conformal invariant. A modification of this construction also gives the Fefferman-Graham conformal invariant [16]. It would be interesting to have some characterization of the tensors and operators which can be generated in this way. It is possible that in some sense this is all the conformally invariant ones.

The physical significance of these curved analogues of the homogeneous differential operators is unclear except for the massless field operators (as in the Introduction). In this context, the fact that the curved generalized deR ham sequence is no longer a complex is normally referred to as the existence of Buchdahl conditions (see e.g. [27]).

\section{References}

1. Baston, R. J.: The algebraic construction of invariant differential operators. D.Phil. thesis, Oxford University 1985

2. Bateman, H.: The transformation of the electrodynamical equations. Proc. L. M. S. 8, 223-264 (1910)

3. Bernstein, I. N., Gelfand, I. M., Gelfand, S. I.: Differential operators on principal affine spaces and investigation of $\mathrm{g}$-modules. In: Lie groups and their representations, Summer School of Bolyai János Math. Soc. Gelfand, I. M. (ed), pp. 21-64. London: Hilgar 1975

4. Boe, B. D., Collingwood, D. H.: A comparison theory for the structure of induced representations. J. Algebra 94, 511-545 (1985)

5. Boe, B. D., Collingwood, D. H.: A comparison theory for the structure of induced representations II. Math. Z. 190, 1-11 (1985)

6. Bott, R.: Homogeneous vector bundles. Ann. Math. 66, 203-248 (1957)

7. Cunningham, E.: The principal of relativity in electrodynamics and an extension thereof. Proc. L. M. S. 8, 77-98 (1910)

8. Dixmier, J.: Enveloping algebras. Amsterdam: North Holland 1977

9. Dubrovin, B. A., Fomenko, A. T., Novikov, S. P.: Modern geometry, methods and applications. Moscow: Nauka 1979

10. Eastwood, M. G., Penrose, R., Wells, R. O., Jr.: Cohomology and massless fields. Commun. Math. Phys. 78, 305-351 (1981)

11. Eastwood, M. G., Tod, K. P.: Edth-a differential operator on the sphere. Math. Proc. Camb. Phil. Soc. 92, 317-330 (1982)

12. Eastwood, M. G.: Complexification, twistor theory, and harmonic maps of Riemann surfaces. Bull. A. M. S. 11, 317-328 (1984)

13. Eastwood, M. G.: The generalized Penrose-Ward transform. Math. Proc. Camb. Phil. Soc. 97, 165187 (1985)

14. Eastwood, M. G.: A duality for homogeneous bundles on twistor space. J. L. M. S. 31, 349-356 (1985)

15. Eastwood, M. G., Singer, M. A.: A conformally invariant Maxwell gauge. Phys. Lett. 107A, 73-74 (1985) 
16. Fefferman, C., Graham, C. R.: Conformal invariants. Preprint, Princeton University 1984

17. Fegan, H. D.: Conformally invariant first order differential operators. Q. J. Math. 27, 371-378 (1976)

18. Harish Chandra: Some applications of the universal enveloping algebra of a semi-simple Lie algebra. Trans. A. M. S. 70, 28-99 (1951)

19. Humphreys, J. E.: Introduction to Lie algebras and representation theory. Grad. Text Math. 9, Berlin, Heidelberg, New York: Springer 1972

20. LeBrun, C. R.: Spaces of complex null geodesics in complex-Riemannian geometry. Trans. A. M. S. 278, 209-231 (1983)

21. Lepowsky, J.: A generalization of the Bernstein-Gelfand-Gelfand resolution. J. Algebra 49, 496-511 (1977)

22. Penrose, R.: Zero-rest-mass fields including gravitation: asymptotic behaviour. Proc. R. Soc. Lond. A248, 159-203 (1965)

23. Penrose, R.: Twistor algebra. J. Math. Phys. 8, 345-366 (1967)

24. Penrose, R.: The structure of space-time. In: Battelle Rencontres 1967, pp. 121-235. New York: Benjamin 1968

25. Penrose, R.: Twistor theory, its aims and achievements. In: Quantum gravity: an Oxford Symposium. Isham, C. J., Penrose, R., Sciama, D. W. (eds.). pp. 267-407. Oxford: Clarendon Press 1975

26. Penrose, R., Ward, R. S.: Twistors for flat and curved space-time. In: General relativity and gravitation, Vol. II Held, A. (ed.). pp. 283-328. New York, London: Plenum Press 1980

27. Penrose, R., Rindler, W.: Spinors and space-time, Vol. I. Cambridge: Cambridge University Press 1984

28. Penrose, R., Rindler, W.: Spinors and space-time, Vol. II. Cambridge: Cambridge University Press 1986

29. Spencer, D. C.: Overdetermined systems of linear partial differential equations. Bull. A. M. S. 75, 179-239 (1969)

30. Szekeres, P.: Conformal tensors. Proc. R. Soc. Lond. A304, 113-122 (1968)

31. Verma, D. -N.: Structure of certain induced representations of complex semisimple Lie algebras. Ph.D. thesis, Yale University 1966 (cf. Bull. A. M. S. 74, 160-166 (1968))

32. Vogan, D. A.: Representations of real reductive Lie groups. Prog. Math. 15, Boston Basel Stuttgart: Birkhäuser 1981

33. Wells, R. O., Jr.: The conformally invariant Laplacian and the instanton vanishing theorem. In: Seminar on differential geometry. Yau, S. -T. (ed.). pp. 483-498. Ann. Math. Stud. 102, Princeton: Princeton University Press 1982

Communicated by S. -T. Yau

Received June 9, 1986 